02

\title{
Исследование последовательности импульсов динамической развязки для дипольно-связанных спиновых систем с неоднородным уширением*
}

\author{
(C) М.М. Миннегалиев ${ }^{1}$, Р.В. Урманчеев ${ }^{1}$, В.А. Скребнев ${ }^{2}$, С.А. Моисеев ${ }^{1,2, \text { ฯ }}$ \\ ${ }^{1}$ Казанский квантовый центр, Казанский национальный исследовательский \\ технический университет им. А.Н. Туполева - КАИ, \\ 420111 Казань, Россия \\ ${ }^{2}$ Казанский физико-технический институт им .Е.К. Завойского, ФИЦ Казанский научный центр РАН, \\ 420029 Казань, Россия \\ ؟e-mail: s.a.moiseev@kazanqc.org
}

Поступила в редакцию 24.09. 2018 г.

\begin{abstract}
Проведено теоретическое исследование протокола динамической развязки с применением последовательности резонансных радиочастотных (РЧ) импульсов различной формы к системе дипольно-связанных электронных/ядерных спинов с неоднородным уширением резонансной линии. Обсуждается выбор оптимальных параметров РЧ импульсов для реализации долгоживущей широкополосной квантовой памяти на таких спиновых системах.
\end{abstract}

DOI: $10.21883 /$ OS.2019.01.47045.252-18

\section{Введение}

Квантовая память является важным элементом для построения многокубитового универсального квантового компьютера и оптического квантового репитера квантовых коммуникаций на большие расстояния [1-3]. Одно из главных требований к квантовой памяти - это обеспечение достаточно длительного времени хранения произвольных квантовых состояний, в частности, не меньше времени, необходимого для распределения квантовой запутанности по узлам квантовой сети [4]. В недавней работе [5] было достигнуто время хранения около одной секунды на парах цезия, находящихся при комнатной температуре. Для практического использования большой интерес также вызывают неорганические кристаллы, легированные редкоземельными ионами. Переходы внутри $4 f$-оболочки таких ионов обладают значительным неоднородным уширением линии резонансных переходов и сравнительно большим временем когерентности, что делает их многообещающим кандидатом для реализации широкополосной квантовой памяти [6].

Существенное увеличение времени когерентности редкоземельных ионов в кристаллах может быть достигнуто при использовании методов динамической развязки, впервые предложенных в ядерном магнитном резонансе и основанных на применении специальных последовательностей радиочастотных (РЧ) импульсов $[7,8]$, резонансных выбранным квантовым переходам между ядерными спиновыми подуровнями. Методы динамической развязки показали свою эффективность в усреднении негативного влияния флуктуирующих магнитных

* XIII International Conference on Hole Burning, Single Molecule, and Related Spectroscopies: Science and Applications (HBSM-2018), August 6-12, 2018, Suzdal-Moscow, Russia. полей, сбивающих фазу спиновой когерентности $[9,10]$. В последнее время эти методы были успешно использованы в квантовых вычислениях и квантовой памяти [11-14].

Эффективность применения многоимпульсной динамической развязки сильно зависит от свойств флуктуирующих полей, параметров основного гамильтониана изучаемой системы и от длительности цикла динамической развязки. Недавно в работах $[15,16]$ была предложена новая многоимпульсная последовательность РЧ импульсов для использования в динамическом усреднении флуктуаций в системе электронных/ядерных спинов. Последовательности РЧ импульсов с предложенными параметрами (амплитуда, фаза и число используемых импульсов) образуют периодические циклы возбуждения спин-системы, эффективно и быстро усредняющие влияние неоднородности магнитного поля спин-системы $H_{0}$, которое определяет неоднородное уширение резонансного перехода и диполь-дипольное взаимодействие $H_{1}$ между спинами. Такие многоспиновые системы характерны для протоколов квантовой памяти, основанных на использовании эффекта фотонного/спинового эха.

Как известно, экспериментально невозможно абсолютно точно воспроизвести необходимые параметры последовательности РЧ импульсов, и даже небольшие ошибки в площади импульса могут оказывать существенное влияние при большом числе повторений последовательности [17] (например, в работе [18] одна и та же последовательность повторялась более $10^{4}$ раз). Более того, при наличии неоднородного уширения перехода становится важным учет реальной длительности РЧ импульсов.

В настоящей работе представлены результаты исследования последовательности динамической развяз- 
ки [15], имеющего целью изучить влияние ошибок в задании импульсной площади и конечной длительности РЧ импульсов, которые (в отличие от предлагаемых ранее идеальных $\delta$-импульсов) приводят к нежелательным отклонениям в набеге фазы у различных спинов, а также исследовать возможность улучшения использования рассматриваемой динамической развязки за счет применения импульсов с протяжкой несущей частоты.

\section{Влияние ошибки в определении площади и учет конечной длительности импульса}

Во вращающейся системе координат в неоднородном магнитном поле гамильтониан спиновой системы имеет следующий вид [19]:

$$
H=\sum_{i} \Delta_{i} I_{z}^{i}+\sum_{i>j} a_{i, j}\left(3 I_{z}^{i} I_{z}^{j}+I^{i} I^{j}\right),
$$

где $I_{i}^{x y z}-$ спиновый оператор $i$-го атома, $I^{i} I^{j}=I_{x}^{i} I_{x}^{j}+$ $+I_{y}^{i} I_{y}^{j}+I_{z}^{i} I_{z}^{j}, \Delta_{i}-$ отстройка частоты $i$-го атома, $a_{i j}=\frac{1}{4} \gamma^{2} \hbar^{2} \frac{\left(1-3 \cos ^{2} \theta_{i j}\right)}{r_{i j}^{3}}-$ константа спин-спинового взаимодействия между ядерными спинами $i$ и $j, \gamma-$ гиромагнитное отношения для ядерного спина, $\hbar-$ постоянная Планка, ниже принятая равной единице для простоты описания, $r_{i j}$ - расстояние между спинами $i$ и $j, \theta_{i j}$ - угол между направлением постоянного магнитного поля $\mathbf{H}_{z}$ и вектором $\mathbf{r}_{i j}$.

Для удобства описания действия импульсной последовательности на спиновую систему остановимся на анализе свойств пары взаимодействующих спинов, имеющей, согласно (1), следующий гамильтониан:

$$
H_{12}=\Delta_{1} I_{z}^{1}+\Delta_{2} I_{z}^{2}+a\left(3 I_{z}^{1} I_{z}^{2}+I^{1} I^{2}\right) .
$$

Для нахождения гамильтониана спиновой системы $\widetilde{H}_{12}(t)$ в представлении взаимодействия при воздействии РЧ импульсов последовательности будем использовать следующее соотношение:

$$
\widetilde{H}_{12}(t)=U_{1}^{-1}(t) H_{12} U_{1}(t) .
$$

В работе [15] было показано, что после одного цикла последовательности средний гамильтониан

$$
\begin{aligned}
\bar{H}_{12}= & \widetilde{H}_{12}(0-\tau)+\widetilde{H}_{12}(\tau-2 \tau)+\widetilde{H}_{12}(2 \tau-3 \tau) \\
& +\widetilde{H}_{12}(3 \tau-4 \tau)+\widetilde{H}_{12}(4 \tau-5 \tau)+\widetilde{H}_{12}(5 \tau-6 \tau)
\end{aligned}
$$

усредняется до нуля. Таким образом, в первом порядке происходит динамическое усреднение как дипольдипольного взаимодействия между спинами, так и неоднородности магнитного поля. Однако в экспериментальных условиях невозможно абсолютно точно задать необходимую импульсную площадь РЧ импульса, что ведет

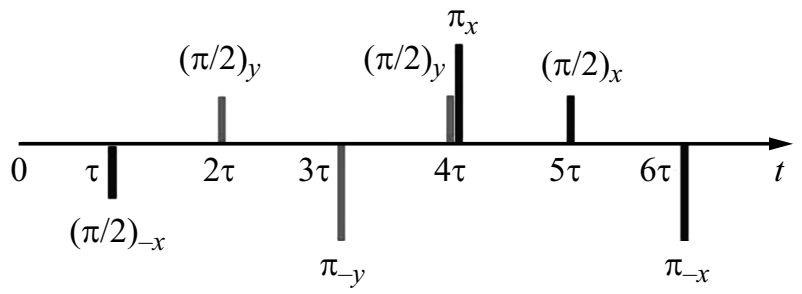

Предложенная последовательность динамической развязки.

к неточному углу поворота вектора намагниченности. Обозначим эту ошибку через малую величину $\varepsilon$.

Мы рассмотрели два варианта данного отклонения. В первом случае $\varepsilon$ представляется постоянной величиной, одинаковой как для $\pi / 2$-, так и для $\pi$-импульса, так что оператор эволюции для первого импульса последовательности $(\pi / 2)_{-x}$ примет вид

$$
U_{1}(\tau-2 \tau)=e^{-i\left(\frac{\pi}{2}+\varepsilon\right) I_{x}}
$$

вместо

$$
U_{1}(\tau-2 \tau)=e^{-i \frac{\pi}{2} I_{x}}
$$

для идеального случая. Если ограничиться только первым порядком в ошибке площади импульса $\varepsilon$, то средний гамильтониан для одного цикла последовательности РЧ импульсов (рисунок) примет вид

$$
\begin{aligned}
\bar{H}_{12}= & -\varepsilon\left\{2 \Delta_{1} I_{1}^{z}+2 \Delta_{2} I_{2}^{z}+\Delta_{1} I_{1}^{x}\right. \\
& \left.+\Delta_{2} I_{2}^{x}+6 a_{12}\left(I_{1}^{z} I_{2}^{y}+I_{2}^{z} I_{1}^{y}\right)\right\} .
\end{aligned}
$$

Во втором случае ошибку поворота вектора намагниченности на углы $\pi / 2$ и $\pi$ обозначим через $\varepsilon$ и $2 \varepsilon$ соответственно. В этом случае средний гамильтониан одного цикла последовательности примет вид

$$
\bar{H}_{12}=-2 \varepsilon\left\{\Delta_{1} I_{1}^{z}+\Delta_{2} I_{2}^{z}+3 a_{12}\left(I_{1}^{z} I_{2}^{y}+I_{2}^{z} I_{1}^{y}\right)\right\} .
$$

В работе [15] рассматривался идеальный случай $\delta$-импульсов (очень коротких и неограниченно интенсивных импульсов). Поэтому стоит учесть ошибку, которая возникает из-за конечного времени длительности импульса. Эта ошибка приводит к различному набегу фазы отдельных спинов из-за неоднородности магнитного поля. В данном случае оператор эволюции примет вид

$$
U_{1}(t)=e^{i\left(\Delta I_{z}-\Omega I_{x, y}\right) \delta t},
$$

где $\Omega$ - частота Раби поворота вектора намагниченности вдоль осей $X$ или $Y$ при действии РЧ импульса с длительностью $\delta t$. Так, для первого импульса последовательности $\left(\frac{\pi}{2}\right)_{-x}$ оператор эволюции примет вид

$$
U_{1}(\tau-2 \tau)=e^{i\left(\Delta t I_{z}-\frac{\pi}{2} I_{x}\right)}
$$

(вместо $U_{1}(\tau-2 \tau)=e^{-i \frac{\pi}{2} I_{x}}$ для $\delta$-импульса), что учитывает зависимость ориентации оси вращения и угла поворота спина от его частотной расстройки. При 
расчете оператора эволюции всей последовательности импульсов, используя разложение Магнуса [20] и ограничиваясь первым членом этого разложения, находим средний гамильтониан цикла

$$
\begin{aligned}
\bar{H}_{12}= & \left(\frac{15 \Delta_{1}^{2}}{2 \Omega}-\frac{2 \Delta_{1}^{2}}{\Omega^{\prime}}\right) I_{1}^{x}+\left(\frac{15 \Delta_{2}^{2}}{2 \Omega}-\frac{2 \Delta_{2}^{2}}{\Omega^{\prime}}\right) I_{2}^{x} \\
& +\left(\frac{4 \Delta_{1}^{2}}{\Omega}-\frac{2 \Delta_{1}^{2}}{\Omega^{\prime}}\right) I_{1}^{y}+\left(\frac{4 \Delta_{2}^{2}}{\Omega}-\frac{2 \Delta_{2}^{2}}{\Omega^{\prime}}\right) I_{2}^{y} \\
& +\left(-\frac{3 \Delta_{1}^{2}}{\Omega}-\frac{\Delta_{1}^{2}}{\Omega^{\prime}}\right) I_{1}^{z}+\left(-\frac{3 \Delta_{2}^{2}}{\Omega}-\frac{\Delta_{2}^{2}}{\Omega^{\prime}}\right) I_{2}^{z} \\
& +3 a_{12}\left[\left(-\frac{4 \Delta_{2}}{\Omega}+\frac{2 \Delta_{2}}{\Omega^{\prime}}+\frac{\Delta_{1}}{\Omega}\right) I_{1}^{x} I_{2}^{z}\right. \\
& +\left(-\frac{4 \Delta_{1}}{\Omega}+\frac{2 \Delta_{1}}{\Omega^{\prime}}+\frac{\Delta_{2}}{\Omega}\right) I_{1}^{z} I_{2}^{x} \\
& +\left(\frac{2 \Delta_{2}}{\Omega}-\frac{2 \Delta_{1}}{\Omega^{\prime}}-\frac{3 \Delta_{1}}{2 \Omega}\right) I_{1}^{x} I_{2}^{y} \\
& +\left(\frac{2 \Delta_{1}}{\Omega}-\frac{2 \Delta_{2}}{\Omega^{\prime}}-\frac{3 \Delta_{2}}{2 \Omega}\right) I_{1}^{y} I_{2}^{x} \\
& +\left(\frac{3 \Delta_{1}}{\Omega}+\frac{3 \Delta_{2}}{\Omega}-\frac{\Delta_{1}}{\Omega^{\prime}}-\frac{\Delta_{2}}{\Omega^{\prime}}\right) I_{1}^{y} I_{2}^{z} \\
& \left.+\left(\frac{3 \Delta_{1}}{\Omega}+\frac{3 \Delta_{2}}{\Omega}-\frac{\Delta_{1}}{\Omega^{\prime}}-\frac{\Delta_{2}}{\Omega^{\prime}}\right) I_{1}^{z} I_{2}^{y}\right] \\
& +\left(\frac{3}{\Omega}\right)
\end{aligned}
$$

где $\Omega$ и $\Omega^{\prime}$ - частоты Раби для $\left(\frac{\pi}{2}\right)-$ и $\pi$-импульсов соответственно.

Как видно из (5), неидеальность РЧ импульсов проявляется возникновением дополнительных локальных полей в среднем гамильтониане, ориентированных в различных направлениях в лабораторной системе координат с углами ориентации, квадратично зависящими от частотных отстроек спинов. Дополнительные поля вызывают ошибку в набеге фазы различных спинов, имеющую вид

$$
\delta \varphi_{1} \sim n \frac{\overline{\Delta^{2}}}{\Omega} \tau_{c}
$$

где $\tau_{c}$ - длительность цикла, $n-$ число циклов, $\overline{\Delta^{2}}-$ среднеквадратичное отклонение частоты возбуждаемых спинов. Кроме того, такие импульсы вызывают появление эффективного диполь-дипольного взаимодействия, пропорционального частотной отстройке спинов, приводящего к дополнительной расфазировке с характерной величиной разброса фаз у различных пар спинов

$$
\delta \varphi_{2} \sim n a_{12} \frac{\sqrt{\overline{\Delta^{2}}}}{\Omega} \tau_{c}
$$

Таким образом, общее влияние эффективного гамильтониана (5) растет с увеличением частотных отстроек, при этом увеличение частоты Раби РЧ импульсов ослабляет оба типа дефазировки спинов. Однако рост числа циклов неизбежно увеличивает негативное влияние неидеальности РЧ импульсов. Существенно, что в фазу $\delta \varphi_{2}$ входит общая ширина линии $\sqrt{\overline{\Delta^{2}}}=\Delta_{i n}$, что означает необходимость уменьшения ее величины. Для более сильного подавления дефазировки в системе спинов с неоднородным уширением линии ниже мы рассматриваем возможность использование импульсов с плавной огибающей и протяжкой несущей частоты, применение которых, как известно, ослабляет зависимость эволюции спина от его частотной расстройки при воздействии таких импульсов [21]. Это позволило экспериментально добиться более точной реализации $\pi$-импульсов на неоднородно уширенном резонансном переходе [22,23].

\section{Прохождение импульса с протяжкой частоты}

Задачу об эволюции спина в РЧ импульсе с протяжкой частоты удобно решать, переходя к амплитудам вероятности собственных состояний двухуровневой системы $C_{1}(t)$ и $C_{2}(t)$ :

$$
|\psi\rangle=C_{1}(t) e^{-i E_{1} t / \hbar}|1\rangle+C_{2}(t) e^{-i E_{2} t / \hbar}|2\rangle,
$$

где $E_{1,2}$ - энергии состояний $|1\rangle$ и $|2\rangle$. В общем случае для описания поведения амплитуд $C_{1}(t)$ и $C_{2}(t)$ справедлива следующая система уравнений:

$$
\left(\begin{array}{l}
\dot{C}_{1} \\
\dot{C}_{2}
\end{array}\right)=\left(\begin{array}{cc}
0 & \frac{i}{2} \dot{A} e^{i B} \\
\frac{i}{2} \dot{A} e^{-i B} & 0
\end{array}\right)\left(\begin{array}{l}
C_{1} \\
C_{2}
\end{array}\right)
$$

где $A$ и $B$ определяются параметрами РЧ импульсов и спина, при этом мы выбираем импульсы в форме гиперболического секанса и протяжкой частоты в форме гиперболического тангенса:

$$
\dot{A}=\frac{\alpha}{\pi \tau} \operatorname{sech} \frac{t-t_{0}}{\tau}, \quad \dot{B}=\frac{1}{\pi \tau}\left[\Delta+\beta \tanh \frac{\left(t-t_{0}\right)}{\tau}\right],
$$

где $\alpha$ и $\tau$ определяют соответственно амплитуду и длительность импульса, а $\Delta$ и $\beta-$ постоянную отстройку и диапазон протяжки частоты, при этом направление оси поворота определяется фазой $B$. Из (7) находим уравнения второго порядка для $C_{1}(t)$ и $C_{2}(t)$ :

$$
\ddot{C}_{1,2}+\left(\mp i \dot{B}-\frac{\ddot{A}}{\dot{A}}\right) \dot{C}_{1,2}+\left(\frac{\dot{A}}{2}\right)^{2} C_{1,2}=0 .
$$

В работе [24] было показано, что после перехода к переменной

$$
z=\frac{1}{2}\left[1+\tanh \left(t-t_{0}\right)\right]
$$

$C_{1}$ и $C_{2}$ подчиняются гипергеометрическому уравнению

$$
z(1-z) \frac{d^{2} C_{1}}{d z^{2}}+[c-(a+b+1)] z \frac{d C_{1}}{d z}-a b C_{1}=0 .
$$


Для $C_{2}$ справедливо аналогичное уравнение, в котором $a, b, c \rightarrow a^{*}, b^{*}, c^{*}$, а коэффициенты $a, b, c$ определяются параметрами импульса:

$$
\begin{gathered}
a=\frac{1}{2 \pi}\left[\left(\alpha^{2}-\beta^{2}\right)^{\frac{1}{2}}-i \beta\right], \\
b=\frac{1}{2 \pi}\left[-\left(\alpha^{2}-\beta^{2}\right)^{\frac{1}{2}}-i \beta\right], \\
c=\frac{1}{2}\left(1-i \frac{\Delta-\beta}{\pi}\right) .
\end{gathered}
$$

Здесь исправлена опечатка в [24] в определении $c$.

Используя (10), в работе [24] найден общий вид решения, выражаемого через суперпозицию гипергеометрических функций:

$$
\begin{aligned}
C_{1}= & p_{1} F(a, b, c, z) \\
& \quad+p_{2} z^{1-c} F(a+1-c, b+1-c, 2-c, z), \\
C_{2}= & q_{1} F\left(a^{*}, b^{*}, c^{*}, z\right) \\
& +q_{2} z^{1-c^{*}} F\left(a^{*}+1-c^{*}, b^{*}+1-c^{*}, 2-c^{*}, z\right),
\end{aligned}
$$

и на их основе в работе было исследовано поведение населенности уровней $\left|C_{1}\right|^{2}$ и $\left|C_{2}\right|^{2}$ для изначально невозмущенной системы $\left(C_{1}\left(t_{0}\right)=1, C_{2}\left(t_{0}\right)=0\right)$ при повороте вектора Блоха вокруг оси $X\left(B_{0}=0\right)$. Для нахождения решения при любых начальных условиях $\left(C_{1}\left(t_{0}\right) \neq 0, C_{2}\left(t_{0}\right) \neq 0\right)$ мы исследовали асимптотику решений (12), (13) и нашли общей вид параметров $p_{2}$ и $q_{2}$ :

$$
\begin{gathered}
p_{2}=\frac{i}{2 \pi(1-c)} e^{i\left(B_{0}-\frac{\beta}{\pi} \ln 2\right]} q_{1}, \quad q_{1}=C_{2}\left(t_{0}\right), \\
q_{2}=\frac{i}{2 \pi\left(1-c^{*}\right)} e^{-i\left[B_{0}-\frac{\beta}{\pi} \ln 2\right]} p_{1}, \quad p_{1}=C_{1}\left(t_{0}\right) .
\end{gathered}
$$

Используя решения (12)-(14), мы получаем матрицу эволюции $\hat{U}$, описывающую поведение спина при воздействии РЧ импульса согласно выражению

$$
\left(\begin{array}{l}
C_{1}(t) \\
C_{2}(t)
\end{array}\right)=\hat{U}\left(\begin{array}{l}
C_{1}\left(t_{0}\right) \\
C_{2}\left(t_{0}\right)
\end{array}\right),
$$

с матричными элементами

$$
\begin{aligned}
U_{(11)}= & F(a, b, c, z), \quad U_{(22)}=F\left(a^{*}, b^{*}, c^{*}, z\right), \\
U_{(12)}= & \frac{i \alpha}{2 \pi\left(1-c^{*}\right)} e^{-i\left[B_{0}-\frac{\beta}{\pi} \ln 2\right]} z^{1-c^{*}} \\
& \times F\left(a^{*}+1-c^{*}, b^{*}+1-c^{*}, 2-c^{*}, z\right), \\
U_{(21)}= & \frac{i \alpha}{2 \pi\left(1-c^{*}\right)} e^{-i\left[B_{0}-\frac{\beta}{\pi} \ln 2\right]} z^{1-c^{*}} \\
& \times F\left(a^{*}+1-c^{*}, b^{*}+1-c^{*}, 2-c^{*}, z\right) .
\end{aligned}
$$

При этом произвольные значения импульсной площади и направление магнитного поля РЧ импульса задаются выбором параметров $\alpha$ и $B_{0}$. Используя найденное выражение для $\hat{U}$ взамен матриц типа $e^{i\left(\Delta \delta t I_{z}-\frac{\pi}{2} I_{x}\right)}$, использованных ранее, можно найти соответствующие им матрицы для воздействия импульсов со свипированной частотой и построить общей оператор эволюции для всей многоимпульсной последовательности и проанализировать возможность подавления дефазировки $\delta \varphi_{1}$ и $\delta \varphi_{2}$ на поведение спинов в изучаемой задаче, что является предметом последующего детального изучения.

\section{Заключение}

В работе представлено исследование влияния неидеальных параметров последовательности импульсов динамической развязки [15] для системы дипольно-связанных двухуровневых атомов или спинов с неоднородным уширением линии. Учет конечной длительности используемых импульсов последовательности показал наличие двух типов дефазировок: одночастичной $\delta \varphi_{1}$ и двухчастичной $\delta \varphi_{2}$, величина которых пропорциональна второй и первой степеням ширины линии взаимодействующих спинов. Также была получена оценка влияния ошибки в определении площади отдельного импульса на гамильтониан системы многоимпульсной последовательности.

Мы нашли матрицу эволюции спинов при воздействии РЧ импульса с плавной огибающей и протяжкой несущей частоты. Использование таких импульсов представляется наиболее перспективным для применения в изучаемой многоимпульсной последовательности.

Работа поддержана грантом Российского научного фонда № 14-12-01333 П.

\section{Список литературы}

[1] Briegel H.-J., Dür W., Cirac J.I., Zoller P. // Phys. Rev. Lett. 1998. V. 81. N 26. P. 5932.

[2] Heshami K. et. al. // J. Mod. Opt. 2016. V. 63. N 20. P. 2005.

[3] Tittel W. et. al. // Laser Photon. Rev. 2009. V. 4. N 2. P. 244.

[4] Sangouard N., Simon C., Riedmatten H., Gisin N. // Rev. Mod. Phys. 2011. V. 83. N 1. P. 33.

[5] Katz O., Firstenberg O. // Nat. Commun. 2018. V. 9. P. 2074.

[6] Thiel C.W., Bottger T., Cone R.L. // J. Luminesc. 2011. V. 131. N 3. P. 353.

[7] Paz-Silva G.A., Lee S.W., Green T.J., Viola L. // New J. Phys. 2016. V. 18. N 7. P. 073020.

[8] Genov G.T., Schraft D., Vitanov N.V, Halfmann T. // Phys. Rev. Lett. 2017. V. 118. N 13. P. 1.

[9] Lim H.J. et. al. // Phys. Rev. B. 2018. V. 97. N 6. P. 1.

[10] Pascual-Winter M.F., Tongning R.-C., Chaneliére T., Le Gouët J.-L. // Phys. Rev. B. 2012. V. 86. P. 184301.

[11] Viola L., Knill E., Lloyd S. // Phys. Rev. Lett. 1999. V. 82. N 12. P. 2417.

[12] West J.R., Fong B.H., Lidar D.A. // Phys. Rev. Lett. 2010. V. 104. P. 130501. 
[13] Yang W., Wang Z., Liu R. // Front. Phys. China. 2011. V. 6. N 1. P. 2.

[14] Maurer P.C. et. al. // Science. 2012. V. 336. N 6086. P. 1283.

[15] Moiseev S.A. Skrebnev V.A. // Phys. Rev. A. 2015. V. $91 . \mathrm{N} 2$. P. 022329.

[16] Moiseev S.A., Skrebnev V.A. // J. Phys. B. At. Mol. Opt. Phys. 2015. V. 48. N 13. P. 135503.

[17] Souza A.M., Ävarez G.A., Suter D. // Phys. Rev. Lett. 2011. V. 106. N 24. P. 1.

[18] Zhong M. et. al. // Nature. 2015. V. 517. N 7533. P. 177.

[19] Slichter C.P. Principles of Magnetic Resonance. Berlin: Springer, 1996.

[20] Magnus W. // Commun. Pure Appl. 1954. V. VII. P. 649.

[21] De Seze F. et. al. // Eur. Phys. J. D. 2005. V. 33. N 3. P. 343.

[22] Gerasimov K.I. et. al. // Opt. Spectrosc. 2017. V. 123. N 2. P. 200.

[23] Damon V. et. al. // New J. Phys. 2011. V. 13. N 9. P. 093031.

[24] Hioe F.T. // Phys. Rev. A. 1984. V. 30. N 4. P. 2100. 